\title{
Effect of temperature on the effective mass and the neutron skin of nuclei
}

\author{
E. Yüksel ${ }^{1}$, E. Khan ${ }^{2}$, K. Bozkurt ${ }^{1}$, and G. Colò ${ }^{3}$ \\ ${ }^{1}$ Physics Department, Yildiz Technical University, 34220 Esenler, \\ Istanbul, Turkey \\ ${ }^{2}$ Institut de Physique Nucléaire, Université Paris-Sud, \\ IN2P3-CNRS, F-91406 Orsay Cedex, France \\ ${ }^{3}$ Dipartimento di Fisica, Università degli Studi and INFN, Sezione \\ di Milano, 20133 Milano, Italy
}

July 16, 2018

\begin{abstract}
We study the finite temperature Hartree-Fock-BCS approximation for selected stable Sn nuclei with zero-range Skyrme forces. Hartree Fock BCS approximation allows for a straightforward interpretation of the results since it involves $u$ and $v$ 's which are not matrices as in HFB. Pairing transitions from superfluid to the normal state are studied with respect to the temperature. The temperature dependence of the nuclear radii and neutron skin are also analyzed. An increase of proton and neutron radii is obtained in neutron rich nuclei especially above the critical temperature. Using different Skyrme energy functionals, it is found that the correlation between the effective mass in symmetric nuclear matter and the critical temperature depends on the pairing prescription. The temperature dependence of the nucleon effective mass is also investigated, showing that proton and neutron effective masses display different behavior below and above the critical temperature, due to the small temperature dependence of the density.
\end{abstract}

\section{Introduction}

Pairing correlations in open-shell nuclei are a crucial part of the mean field calculations which have been investigated over the years and taken into account within either Hartree Fock+BCS or HFB approximations at zero temperature. Over the last decades, the effect of temperature on mean field results has drawn attention due to the vanishing of pairing properties and the phase change of finite nuclei from superfluid to the normal state at critical temperatures. Extensions 
of the mean field models to the finite temperature case have been given in Refs. [1-3]. Thereafter, theoretical investigations have been undertaken with different models and interactions to investigate the relationship between pairing correlations and temperature.

First studies in hot finite nuclei were performed with Hartree-Fock-BCS model $[1,2]$. It has been shown that pairing correlations disappear and nuclei undergo a sharp transition from superfluid to normal state at a critical temperature. Furthermore, the critical temperature and the pairing gap value at zero temperature follow the $T_{c} \simeq 0.567 \Delta_{T=0}$ equation [1]. In Ref [4], similar calculations have been performed with a correction term in the nuclear energy density functional. Pairing transitions in finite nuclei were also predicted within the finite temperature HFB models in Refs. [5-9]. Recently, the effect of different types of pairing interactions on the critical temperature and phase transition of Tin nuclei was investigated within FT-HFB approximation with zero-range Skyrme interaction. It was shown that the pairing gap value is sensitive to the type of the pairing force just below critical temperatures and the critical temperature follows the $T_{c} \simeq 0.5 \Delta_{T=0}$ equation [10]. Very recently, the relationship between pairing and temperature effects was also investigated within the relativistic mean field (RMF) framework in even-even $\mathrm{Ca}, \mathrm{Ni}, \mathrm{Sn}$ and $\mathrm{Pb}$ isotopes in which the critical temperature follows the $T_{c}=0.6 \Delta_{T=0}$ relation [11]. Although the investigation of temperature effect on the pairing properties of nuclei is limited on the experimental side, some experimental signatures were also obtained from the level density measurements of the rare earth ${ }^{161,162} \mathrm{Dy}$, ${ }^{171,172} \mathrm{Yb}$ nuclei. The observed S-shapes in the semi-experimental heat capacities of ${ }^{161,162} \mathrm{Dy},{ }^{171,172} \mathrm{Yb}$ [12] and ${ }^{166,167} \mathrm{Er}$ [13] nuclei were interpreted as the phase change of nuclei from superfluid to the normal state. In addition, the critical temperature at which the pairing correlations are disappearing was found at around $0.5 \mathrm{MeV}$.

Finite temperature effects are also important for astrophysical events, e.g., the reaction rates, r-process nucleosynthesis and core-collapse supernovae. Investigation of the superfluidity and specific heat in the inner crust matter of neutron stars have also been performed within the finite temperature HFB framework in Refs. $[14,15]$. It was shown that the temperature has an impact on the results and should be included in the calculations. Furthermore, the effect of temperature on the effective mass of nucleons, level density and symmetry energy is significant in the supernovae and electron capture rates which takes place at around $1<T<2 \mathrm{MeV}$ [16-20]. Due to the influence of the temperature on the proton and neutron effective mass behavior and its connection with astrophysical events, effect of temperature on nuclei deserves further study. In this framework, the temperature sensitivity of the effective $\mathrm{k}$-mass is revealed for the first time in a microscopic approach.

In the present work, fully self consistent calculations are performed within the finite temperature HFBCS (FT-HFBCS) framework using several types of Skyrme forces. Since we deal with $u$ and $v$ 's in the HFBCS, the interpretation of the temperature effect is more straightforward when compared with HFB approximation. In Section 2, extension of the Hartree Fock BCS model to 
the finite temperature case is presented. In Section 3, the phase change from superfluid to the normal state and effect of temperature on the neutron skin are analyzed. The relation between the effective mass in symmetric nuclear matter and critical temperature is investigated with different Skyrme energy density functionals. Temperature dependence of the effective nucleon masses are also analyzed and discussed. Finally, the conclusion is given in Section 4.

\section{Microscopic model: Finite temperature ex- tension of Hartree Fock BCS approximation}

The BCS approach in nuclei is well known [21] and extensively studied over the years. In the Skyrme-HFBCS approach, the expectation value of the Hamiltonian is obtained as:

$$
E=\left\langle B C S\left|H^{\prime}\right| B C S\right\rangle=E\left[\rho, \kappa, \kappa^{*}\right]
$$

and the total energy density functional of nuclei is given by

$$
\begin{aligned}
E\left[\rho, \kappa, \kappa^{*}\right] & =T+E_{\text {Skyrme }}[\rho]+E_{\text {Coul. }}\left[\rho_{p}\right]+E_{\text {pair }}\left[\rho, \kappa, \kappa^{*}\right] \\
& =\int d^{3} \mathbf{r}\left[T(\mathbf{r})+\mathcal{E}_{\text {Skyrme }}(\mathbf{r})+\mathcal{E}_{\text {Coul. }}(\mathbf{r})+\mathcal{E}_{\text {pair }}(\mathbf{r})\right]
\end{aligned}
$$

Skryme energy density functional for even-even systems is defined as:

$$
\begin{aligned}
\mathcal{E}_{\text {Skyrme }}(\mathbf{r}) & =\frac{1}{2} t_{0}\left[\left(1+\frac{x_{0}}{2}\right) \rho^{2}-\left(x_{0}+\frac{1}{2}\right) \sum_{q} \rho_{q}^{2}\right] \\
& +\frac{t_{1}}{4}\left[\left(1+\frac{x_{1}}{2}\right)\left(\rho \tau+\frac{3}{4}(\nabla \rho)^{2}\right)-\left(x_{1}+\frac{1}{2}\right) \sum_{q}\left(\rho_{q} \tau_{q}+\frac{3}{4}\left(\nabla \rho_{q}\right)^{2}\right)\right] \\
& +\frac{t_{2}}{4}\left[\left(1+\frac{x_{2}}{2}\right)\left(\rho \tau-\frac{1}{4}(\nabla \rho)^{2}\right)+\left(x_{2}+\frac{1}{2}\right) \sum_{q}\left(\rho_{q} \tau_{q}-\frac{1}{4}\left(\nabla \rho_{q}\right)^{2}\right)\right] \\
& -\frac{1}{16}\left(t_{1} x_{1}+t_{2} x_{2}\right) J^{2}+\frac{1}{16}\left(t_{1}-t_{2}\right) \sum_{q} J_{q}^{2} \\
& +\frac{1}{12} t_{3} \rho^{\gamma}\left[\left(1+\frac{x_{3}}{2}\right) \rho^{2}-\left(x_{3}+\frac{1}{2}\right) \sum_{q} \rho_{q}^{2}\right] \\
& +\frac{1}{2} W_{0}\left(J \nabla \rho+\sum_{q} J_{q} \nabla \rho_{q}\right) .
\end{aligned}
$$

In this expression $q$ represents protons (neutrons) and $\rho, J$ and $\tau$ are particle, spin-orbit and kinetic energy densities, respectively. In the extension of the Hartree-Fock-BCS model to the finite temperature case, temperature dependent Fermi-Dirac distribution function is used : 


$$
f_{i}=\left[1+\exp \left(E_{i} / k_{B} T\right)\right]^{-1},
$$

where $E_{i}$ is quasiparticle energy, $k_{B}$ is the Boltzmann constant and $\mathrm{T}$ is the temperature. In the finite temperature case, BCS equations keep the same structure. However, the occupation factor $v_{i}^{2}$ is changed by

$$
v_{i}^{2}\left(1-f_{i}\right)+u_{i}^{2} f_{i}
$$

which also modifies the normal (particle, spin and kinetic energy) and abnormal densities. Such thermal averaged particle densities are given by $[2,14]$,

$$
\begin{aligned}
\rho_{T}(\mathbf{r})= & \frac{1}{4 \pi} \sum_{i}\left(2 j_{i}+1\right)\left[v_{i}^{2}\left(1-f_{i}\right)+u_{i}^{2} f_{i}\right]\left|\phi_{i}(\mathbf{r})\right|^{2}, \\
J_{T}(\mathbf{r})= & \frac{1}{4 \pi} \sum_{i}\left(2 j_{i}+1\right)\left[j_{i}\left(j_{i}+1\right)-l_{i}\left(l_{i}+1\right)-\frac{3}{4}\right] \\
& \times\left\{v_{i}^{2}\left(1-f_{i}\right)+u_{i}^{2} f_{i}\right\}\left|\phi_{i}(\mathbf{r})\right|^{2}, \\
\tau_{T}(\mathbf{r})= & \frac{1}{4 \pi} \sum_{i}\left(2 j_{i}+1\right)\left[v_{i}^{2}\left(1-f_{i}\right)+u_{i}^{2} f_{i}\right] \\
& \times\left[\left(\frac{d \phi_{i}(\mathbf{r})}{d r}-\frac{\phi_{i}(\mathbf{r})}{r}\right)^{2}+\frac{l_{i}\left(l_{i}+1\right)}{r^{2}}\left|\phi_{i}(\mathbf{r})\right|^{2}\right] \\
\kappa_{T}(\mathbf{r})= & -\frac{1}{4 \pi} \sum_{i}\left(2 j_{i}+1\right) u_{i} v_{i}\left(1-2 f_{i}\right)\left|\phi_{i}(\mathbf{r})\right|^{2},
\end{aligned}
$$

where $v_{i}$ and $u_{i}$ are the BCS variational parameters and $\phi_{i}(\mathbf{r})$ the single particle wave function. The Finite temperature Hartree-Fock BCS equations for the Skyrme interaction are obtained with the variation of the expectation value of $H^{\prime}$ :

$$
\delta\left\langle B C S\left|H^{\prime}\right| B C S\right\rangle=0 .
$$

In coordinate space, Hartree Fock equation is written as

$$
\left\{-\nabla \frac{\hbar^{2}}{2 m^{*}(\mathbf{r})} \nabla+U(\mathbf{r})+W \frac{1}{i}(\nabla \times \sigma)\right\} \phi_{i}(\mathbf{r})=\varepsilon_{i} \phi_{i}(\mathbf{r}) .
$$

In our calculations, the pairing correlations are taken into account with a zero-range density-dependent pairing interaction [22]

$$
\begin{aligned}
V_{\text {pair }}\left(\mathbf{r}_{1}, \mathbf{r}_{2}\right) & =V_{0}\left(1-\frac{\rho(\mathbf{r})}{\rho_{0}}\right) \delta\left(\mathbf{r}_{1}-\mathbf{r}_{2}\right) \\
& =V_{\text {eff }}(\rho(\mathbf{r})) \delta\left(\mathbf{r}_{1}-\mathbf{r}_{2}\right),
\end{aligned}
$$

where $V_{0}$ is the pairing strength, $\rho(\mathbf{r})$ is the particle density and $\rho_{0}=0.16$ $\mathrm{fm}^{-3}$ is the nuclear saturation density. All calculations are carried out with the 
surface-type pairing interaction. In order to provide a suitable pairing interaction, the pairing strength $V_{0}$ is fixed for each nuclei according to the $\Delta=12 / \sqrt{A}$ $\mathrm{MeV}$ phenomenological rule. The pairing field is given by

$$
\Delta_{T}(\mathbf{r})=\frac{V_{e f f}(\rho(\mathbf{r}))}{2} \kappa_{T}(\mathbf{r}) .
$$

In addition, the particle number and pairing energy are also impacted with the temperature dependent Fermi-Dirac function. The particle number is defined as

$$
N_{q, T}=\sum_{i}\left(2 j_{i}+1\right)\left[v_{i}^{2}\left(1-f_{i}\right)+u_{i}^{2} f_{i}\right]
$$

and the pairing energy is

$$
E_{\text {pair }, T}=-\frac{\Delta_{T}(\mathbf{r})}{2} u_{i} v_{i}(2 j+1) .
$$

In the following study, it is relevant to derive the effective nucleon mass $\left(m_{q}^{*}\right)$ in terms of the nucleon densities [23]

$$
\begin{aligned}
\frac{\hbar^{2}}{2 m_{q}^{*}}= & \frac{\hbar^{2}}{2 m}+\frac{1}{4}\left[t_{1}\left(1+\frac{x_{1}}{2}\right)+t_{2}\left(1+\frac{x_{2}}{2}\right)\right] \rho \\
& +\frac{1}{8}\left[t_{2}\left(1+2 x_{2}\right)-t_{1}\left(1+2 x_{1}\right)\right] \rho_{q} .
\end{aligned}
$$

All calculations are performed in a $20 \mathrm{fm}$ box with the assumption of spherical symmetry. The cut-off energy is determined with certain number of states around the Fermi energy. In our calculations, the pairing window (the number of levels above the Fermi level) is taken as 20 for both protons and neutrons. The energy difference between the Fermi and the last considered level is around $15 \mathrm{MeV}$ which is large enough to take care of the occupation of levels due to temperature effects.

\section{Results}

In this work, we performed FT-HFBCS calculations using zero-range Skyrme forces. Average neutron pairing gap is calculated via [24]:

$$
\left\langle\Delta_{n}\right\rangle_{\kappa}=\frac{\int d \mathbf{r} \kappa_{T}(\mathbf{r}) \Delta_{T, n}(\mathbf{r})}{\int d \mathbf{r} \kappa_{T}(\mathbf{r})}
$$

Calculations are performed for temperatures up to $1.5 \mathrm{MeV}$ to avoid occupation of unbound single particle levels and additional contributions from neutron vapour [25]. 


\subsection{Tin isotopes}

In panels (a) and (b) of Figure 1, the average neutron pairing gap is plotted as a function of temperature for ${ }^{120} \mathrm{Sn}$ and ${ }^{112} \mathrm{Sn}$ nuclei using various Skyrme interactions. The critical temperature for ${ }^{120} \mathrm{Sn}$ and ${ }^{112} \mathrm{Sn}$ nuclei are found at around $0.65 \mathrm{MeV}$. With the increase of the temperature, pairing correlations are destroyed below the Fermi level. As a result of the high temperature, Cooper pairs are broken and particles are excited to the higher energy levels which leads to the disappearance of pairing correlations [26].
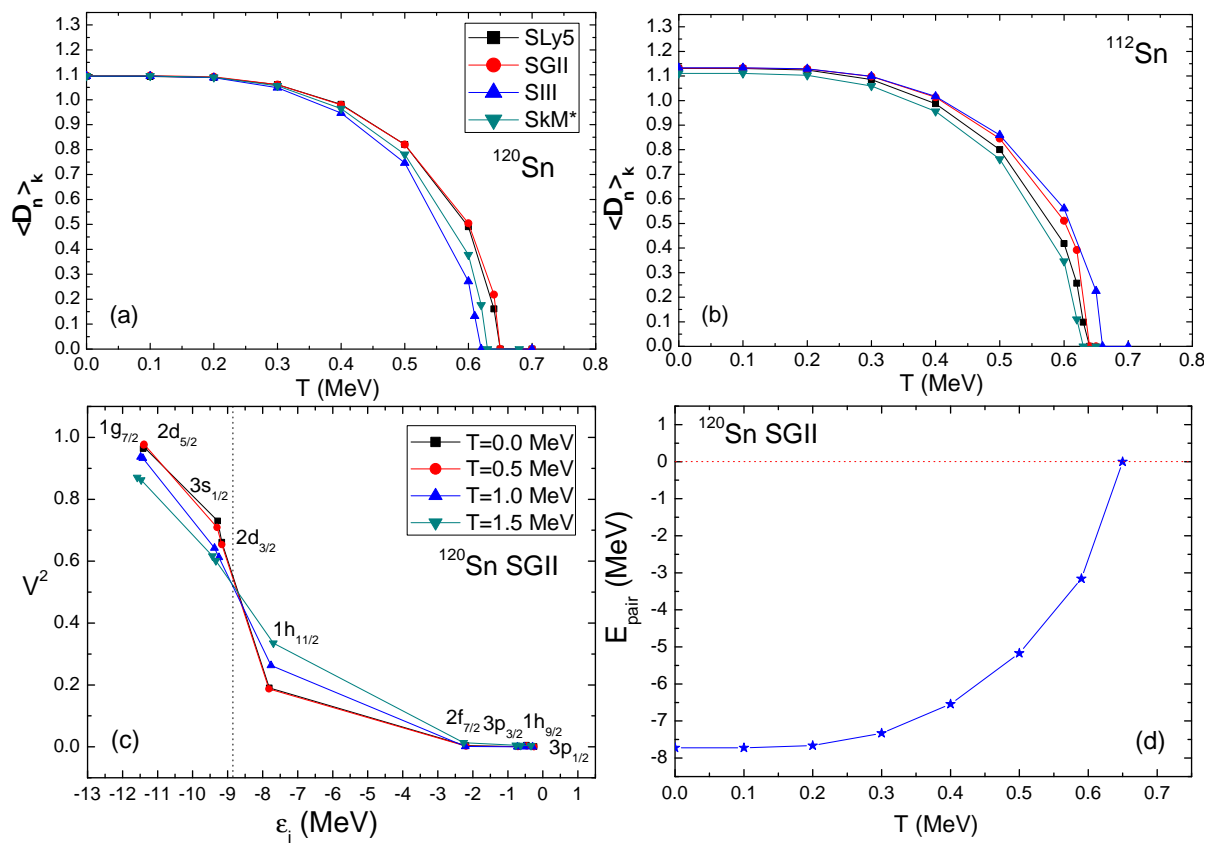

Figure 1: Mean value of the neutron pairing gap as a function of the temperature for ${ }^{120} \mathrm{Sn}$ (a) and ${ }^{112} \mathrm{Sn}$ nuclei (b), occupation probabilities for neutrons as a function of temperature around the Fermi level (c). In ${ }^{120} \mathrm{Sn}$, the dotted line represents the Fermi level at $\mathrm{T}=0.0 \mathrm{MeV}$. Pairing energy as a function of temperature (d).

For the Skyrme interactions used (SLy5 [27], SGII [28], SIII [29], SkM* [30]), the critical temperature and the pairing gap value at zero temperature follow the $T_{c} \simeq 0.57 \Delta_{T=0}$ rule. This is also consistent with the finite temperature HFB results in Ref. [10] in which the critical temperature was found around $0.7 \mathrm{MeV}$ for the ${ }^{124}$ Sn nucleus with SLy4 interaction. Calculations are also performed in $A=106$ to $A=124$ even-even Tin isotopes and similar results are obtained.

In order to provide a clear explanation for the vanishing pairing mechanism, in panel (c) of Fig. 1 we display the temperature dependence of the occupation probabilities with respect to the single particle energies of the ${ }^{120} \mathrm{Sn}$ nucleus us- 
ing the SGII interaction. The single particle levels are chosen around the Fermi level, which are impacted by temperature. With the increase of the temperature, nucleons are excited to higher energies and occupation probabilities are increased above the Fermi level. Especially, the occupation probability of the $1 h_{11 / 2}$ state just above the Fermi level increases above the critical temperature while the occupation probabilities of $3 s_{1 / 2}$ and $2 d_{3 / 2}$ states are decreasing below the Fermi level. In addition, the change of the pairing energy as a function of temperature is displayed in panel (d) of Fig. 1. Although the decrease of the pairing energy is small up to $0.3 \mathrm{MeV}$, a substantial decrease of the pairing energy is obtained for the temperatures between $0.3 \mathrm{MeV}$ and $0.6 \mathrm{MeV}$. At critical temperature, the pairing energy is exactly zero, showing the vanishing of pairing correlations and interpreted as the phase change from superfluid to the normal state. The sharp transition is due to the limitation of the present approach which corresponds the grand-canonical ensemble [6]. Similar results are also obtained with other Tin isotopes and Skyrme interactions: SLy5, SIII and $\mathrm{SkM}^{*}$.

In panels (a) and (b) of Fig. 2, the change of the proton and neutron radii with respect to the temperature is displayed for the ${ }^{120} \mathrm{Sn}$ nucleus with SGII interaction. The interaction SGII [28] is one of the widely used parameterizations of the Skyrme force. It has been derived from SkM*, by improving the Landau parameters and in particular $G_{0}^{\prime}$. This ensures a good description of spin states like the Gamow-Teller resonance, in addition to the reproduction of ground state masses and radii. The proton radius stays constant up to the critical temperature and then increases linearly. Although a small decrease is obtained in the case of the neutron radius up to the critical temperature, it also increases after critical temperature. The temperature dependence of the neutron skin $\left(R_{n}-R_{p}\right)$ is also presented in the panels (c) and (d) of Fig. 2. In the case of ${ }^{120} \mathrm{Sn}$ although a small decrease is obtained before the critical temperature, the neutron skin increases of about $3 \%$ at temperatures above 1 $\mathrm{MeV}$. This is due to the increasing population of larger single-particle states with larger angular momentum $(l)$, such as the $1 h_{11 / 2}$ state, as discussed above. Investigation of the effect of $N / Z$ value on the temperature dependence of the neutron skin is also performed in the Tin isotopic chain. In panel (d) of Fig. 2 , the $R_{n}-R_{p}$ difference with respect to $\mathrm{N} / \mathrm{Z}$ value is displayed. It has been known that HFBCS is not suitable for drip line nuclei due to the formation of unphysical neutron gas [31]. Therefore, we limit our calculations up to ${ }^{120} \mathrm{Sn}$ nucleus in this part. Since both proton and neutron radii increase above the critical temperature, the proton radius weakens the formation of neutron skin in less neutron-rich Tin isotopes with the increase of the temperature. 

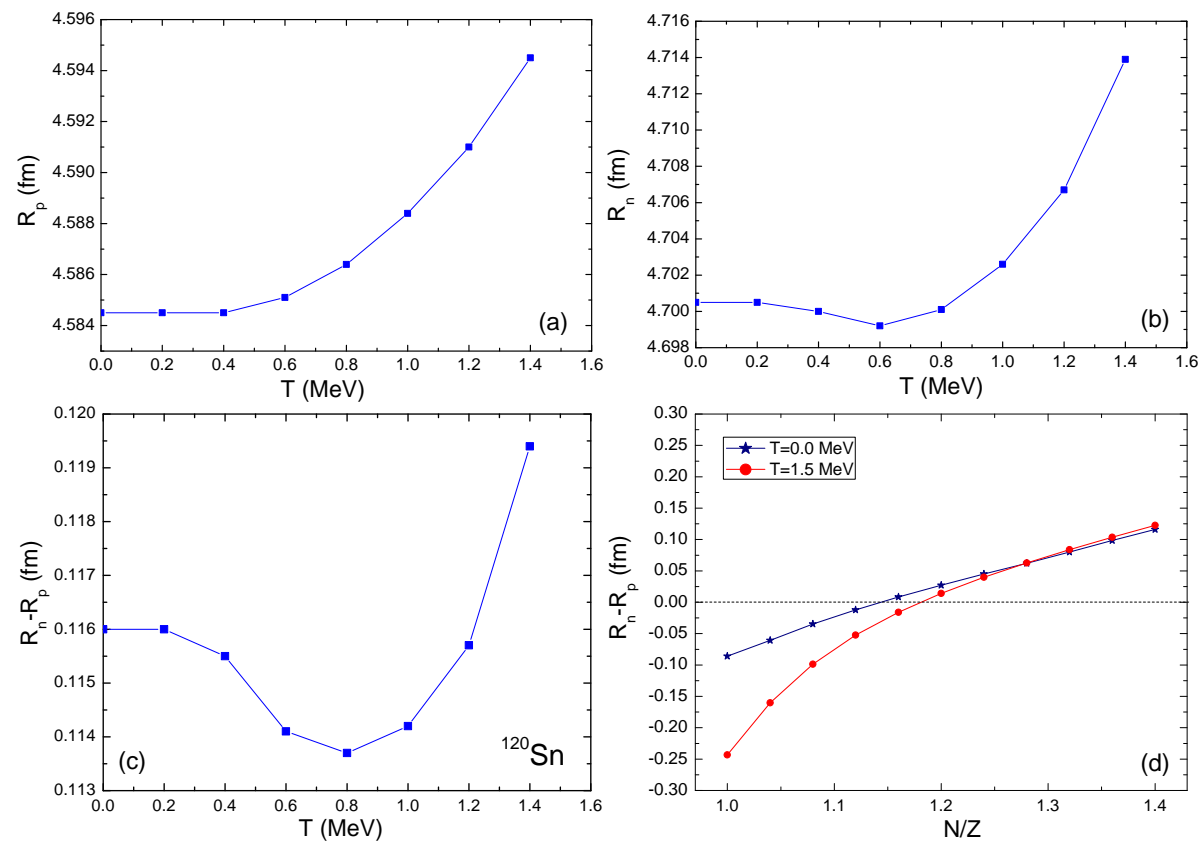

Figure 2: Proton (a) and neutron (b) radius with respect to the temperature for ${ }^{120} \mathrm{Sn}$, calculated with SGII interaction and the change of neutron skin radius of ${ }^{120} \mathrm{Sn}$ nucleus with respect to the temperature (c). $R_{n}-R_{p}$ difference as a function of $\mathrm{N} / \mathrm{Z}$ value for the $\mathrm{Sn}$ isotopes at $\mathrm{T}=0.0 \mathrm{MeV}$ and $\mathrm{T}=1.5 \mathrm{MeV}$ (d).

In order to further investigate the increase of the neutron skin with temperature, in Fig. 3 the neutron partial densities of states $\left(\rho_{n l j, T}(r)\right)$ as a function of temperature are displayed for the ${ }^{120} \mathrm{Sn}$ nucleus. Since temperature impacts mostly the states around the Fermi level, the single neutron levels around the Fermi level are displayed. First, the effect of temperature is seen in the $1 g_{7 / 2}$ single particle level which is below the Fermi level. While the neutron partial densities are decreasing below the Fermi level, an increase in the partial density of the $1 h_{11 / 2}$ state just above the Fermi level is obtained at high temperatures. Proton partial densities also show a similar behavior: while partial densities decrease below Fermi level, an increase is obtained above the Fermi level at high temperatures. This causes an increase in the proton radius. In the case of the $2 f_{7 / 2}, 3 p_{3 / 2}$ and $1 h_{9 / 2}$ neutron states, a more subtle effect is at work: the partial densities decrease up to $\mathrm{T}=1.0 \mathrm{MeV}$ and then increase. The decrease is due to the temperature effects which are destroying the small occupation number generated by pairing effects. Above $1 \mathrm{MeV}$, these states start to be repopulated by temperature effects, because of their increasing Fermi-Dirac factors (feeding from lower energy states). 

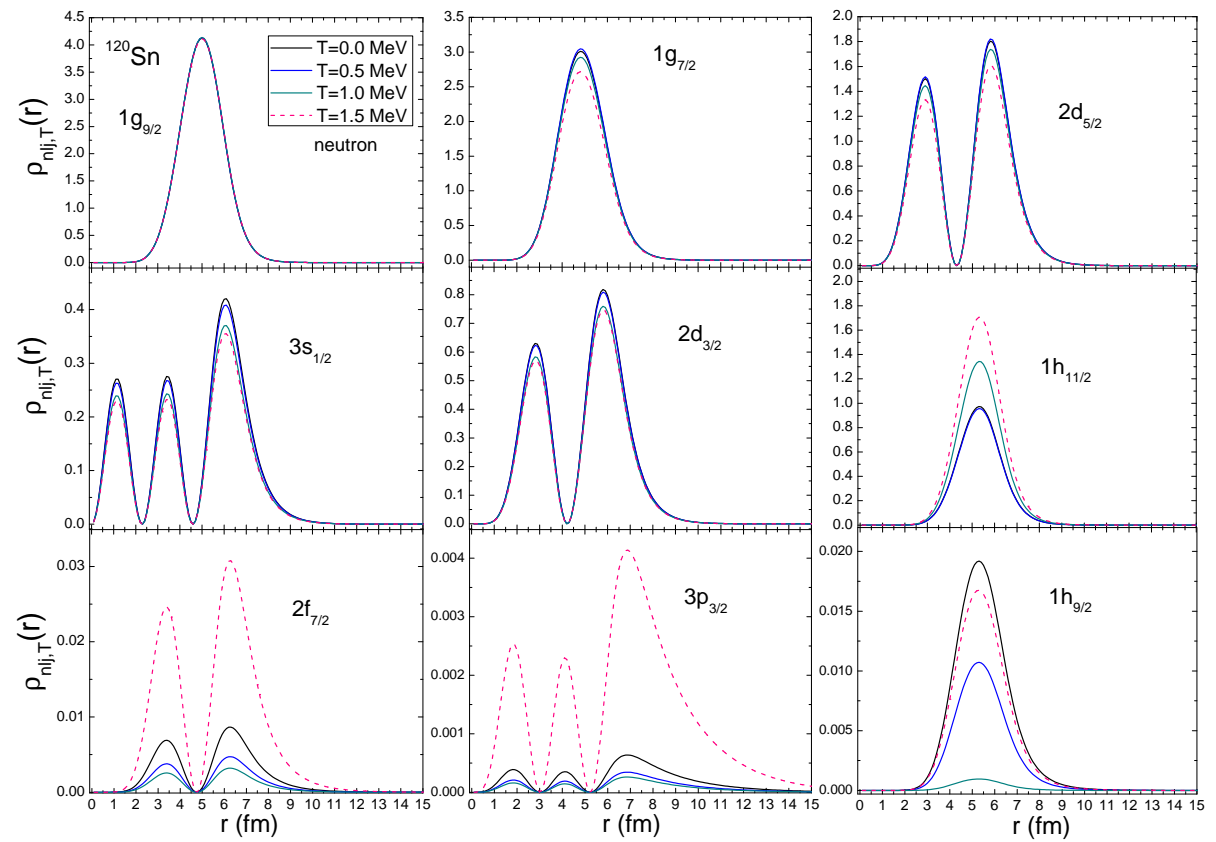

Figure 3: Neutron partial densities of states (see Eq. (6)) as a function of temperature for ${ }^{120} \mathrm{Sn}$ nucleus with SGII interaction. Here, $n, l, j$ are representing the principal, orbital angular momentum and total angular momentum quantum numbers of the corresponding state, respectively.

\subsection{Temperature effects on effective masses}

\subsubsection{Effective mass of symmetric nuclear matter and $T_{c}$}

In this section, our aim is to investigate the possible relation between the effective mass in symmetric nuclear matter (or equivalently $\mathrm{m}^{*}(\mathrm{r}=0)$ ) and the critical temperature. The effective mass is known to impact the level density and pairing correlations which in turn may have an effect on the critical temperature $[18,32]$. In our calculations, $m^{*} / m$ values are ranging from 0.6 to 1.0 for the selected Skyrme energy density functionals.

In symmetric nuclear matter, effective masses for the Skyrme energy density functionals are defined as [27]:

$$
\frac{m_{S k y .}^{*}}{m}=\left[1+\frac{m \rho_{0}}{8 \hbar^{2}}\left\{3 t_{1}+t_{2}\left(5+4 x_{2}\right)\right\}\right]^{-1},
$$

where $\rho_{0}$ is the nuclear saturation density.

The upper panel of Figure 4 shows the influence of the effective mass values of the Skyrme energy density functionals (Eq.(18)) on the critical temperature of ${ }^{120} \mathrm{Sn}$ nucleus. Calculations are performed with 14 different Skyrme interactions, keeping the pairing strength constant, $V_{0}=680 \mathrm{MeV} . \mathrm{fm}^{3}$, for 
all interactions used. A correlation between the effective mass and the critical temperature is obtained, namely with the increase in the effective mass values of the Skyrme interactions the critical temperature increases. Since a larger effective mass generates larger level densities and larger pairing gap values (see lower panel of Fig. 4), the critical temperature at which the pairing correlations are disappearing is also larger. The pairing gap at zero temperature and the critical temperature are strongly correlated by the $T_{c} \simeq 0.57 \Delta_{T=0}$ law and larger effective mass value generates a higher pairing gap value as expected. To check this interpretation, we also plot neutron single particle energies for the ${ }^{120}$ Sn nucleus with SGI and SkX Skyrme interactions (see Fig. 5) which have 0.6 and $1.0 \mathrm{~m}^{*} / \mathrm{m}$ values, respectively. SGI interaction has lower effective mass value and predicts larger energy difference between the $2 d_{3 / 2}$ level and Fermi level (smaller level density).

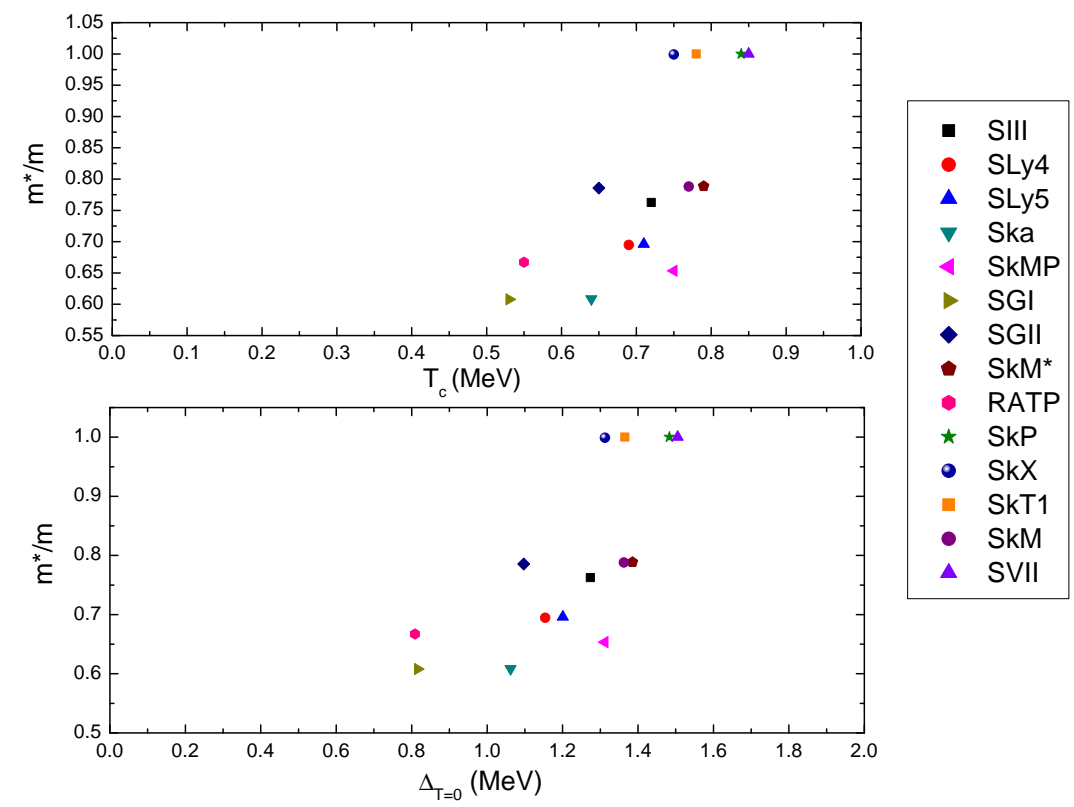

Figure 4: Upper Panel: Effective mass values with respect to the critical temperature. Lower Panel: Effective mass values with respect to the mean value of the pairing gap. Calculations are performed for ${ }^{120} \mathrm{Sn}$ nucleus using 14 different Skyrme interactions. 


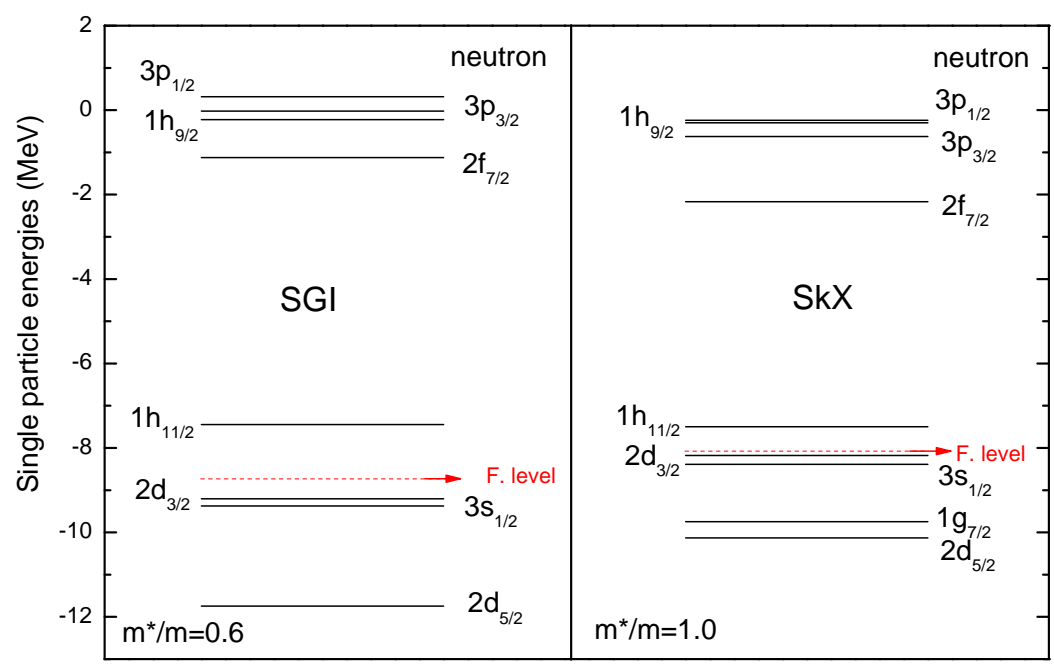

Figure 5: Neutron single particle energies around the Fermi level for ${ }^{120} \mathrm{Sn}$ nucleus with SGI and SkX interactions, at zero temperature.

This low level density around the Fermi level gives smaller pairing gap value. Therefore the critical temperature value for the phase transition is lowered. The SkX interaction has a larger effective mass and predicts smaller energy difference between the $2 d_{3 / 2}$ level and Fermi level. Hence the level densities and pairing gap are enhanced which eventually causes a larger critical temperature value. We checked that same calculations with different Skyrme interactions give similar result.

It should be noted that in the case of imposing a constant pairing gap, the critical temperature is also constant due to the above relation between the pairing gap and the critical temperature. In this case the critical temperature dependence of the effective mass vanishes.

\subsubsection{Temperature dependence of the effective mass in nuclei}

In this section, we investigate the effect of temperature on the effective masses of nuclei. The total effective nucleon mass is obtained with the product of the effective k-mass and $\omega$-mass $\left(m^{*} / m=m_{\omega} m_{k} / m^{2}\right)$. While the k-mass comes from the spatial non-localities and is obtained from the mean field calculations, the $\omega$-mass is related to the frequency dependence of the mean field [16].

The level densities around the Fermi level are affected with the changes in the effective mass as discussed above. Therefore the determination of the temperature dependence of the effective mass is an important task for the level density calculations. The temperature dependence of the effective nucleon mass 
of nuclei have been previously studied in Refs. [16,17,33,34]. The results indicate a decrease of the effective nucleon masses at high temperatures. Therefore the nuclear symmetry energy and electron capture rates in the stellar core collapse, which takes place at 1-2 MeV temperature are also affected [16]. In ref. [17], the temperature dependence of the effective $\omega$-mass was investigated with respect to the temperature. The effective mass was found to decrease which decreases the level density and increases symmetry energy at high temperatures. The changes in the effective proton and neutron masses might have some effects on astrophysical events; for example in the electron capture rates of stellar collapses [17]. Since the rate of the electron capture is closely related with the proton and neutron chemical potentials and consequently with the symmetry energy, an increase or decrease in the effective nucleon masses might lead significant changes in these rates.

In this framework, in the left part of Fig. 6, the effect of temperature on the effective k-masses (at $\mathrm{r}=0$, Eq.(16)) of protons and neutrons are displayed. The results are shown for ${ }^{120} \mathrm{Sn}$ nucleus with SGII interaction.
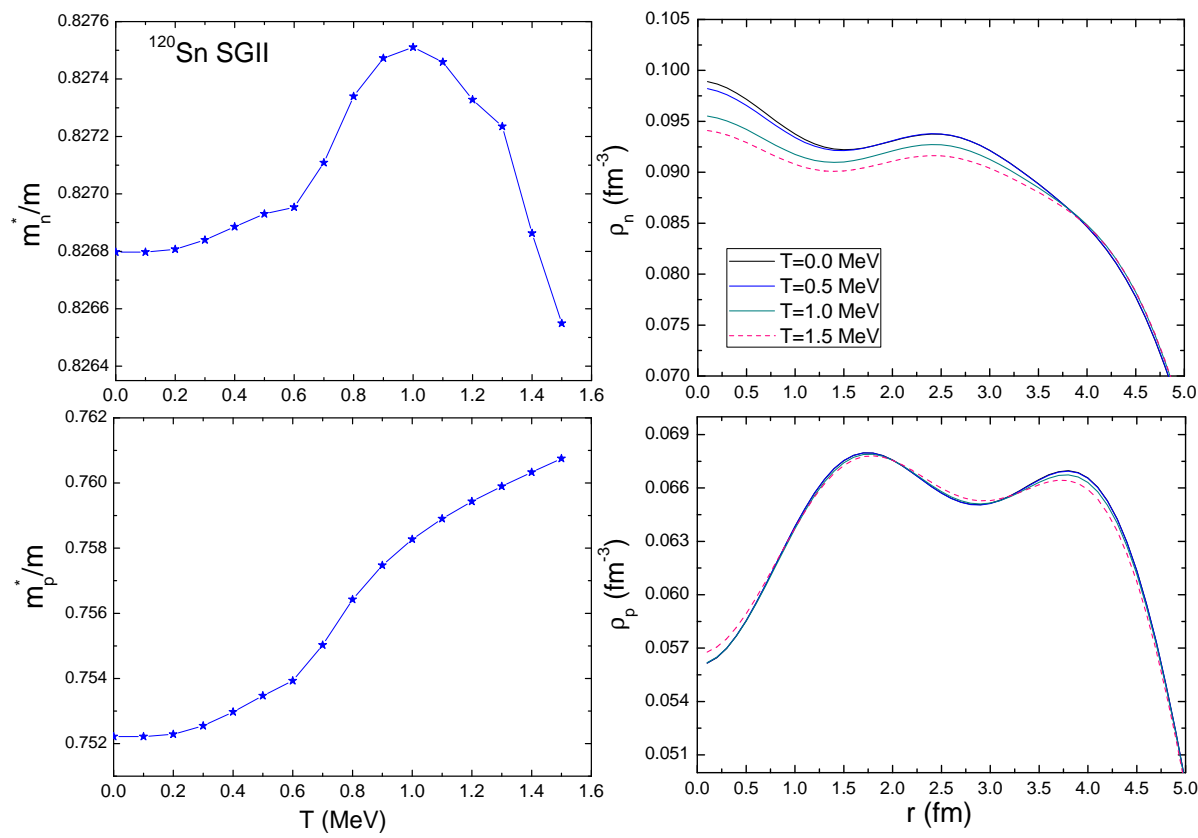

Figure 6: Left Panel: Effect of temperature on the neutron and proton effective masses (at $\mathrm{r}=0$ ) for ${ }^{120} \mathrm{Sn}$ nucleus with SGII interaction. Right Panel: proton and neutron densities with respect to the temperature.

An increase of the effective neutron mass up to $\mathrm{T}=1.0 \mathrm{MeV}$ is observed in the interior of the nuclei. Thereafter, the effective neutron mass suddenly decreases from $\mathrm{T}=1.0 \mathrm{MeV}$ to $\mathrm{T}=1.5 \mathrm{MeV}$. In addition, the increase of the proton effective mass is much more pronounced after the critical temperature. 
The change is about $1.13 \%$ for proton effective mass. Although the changes in the effective masses are small in magnitude as mentioned in previous works [16], it may be relevant to accurately study its behavior. To explain the behavior of the effective proton and neutron masses, we rewrite Eq.(16) using the values of the SGII parameters:

$$
\frac{\hbar^{2}}{2 m_{q}^{*}}=\frac{\hbar^{2}}{2 m}+\left(64.56\left(M e V . f m^{5}\right) \rho-57.67\left(M e V . f m^{5}\right) \rho_{q}\right)
$$

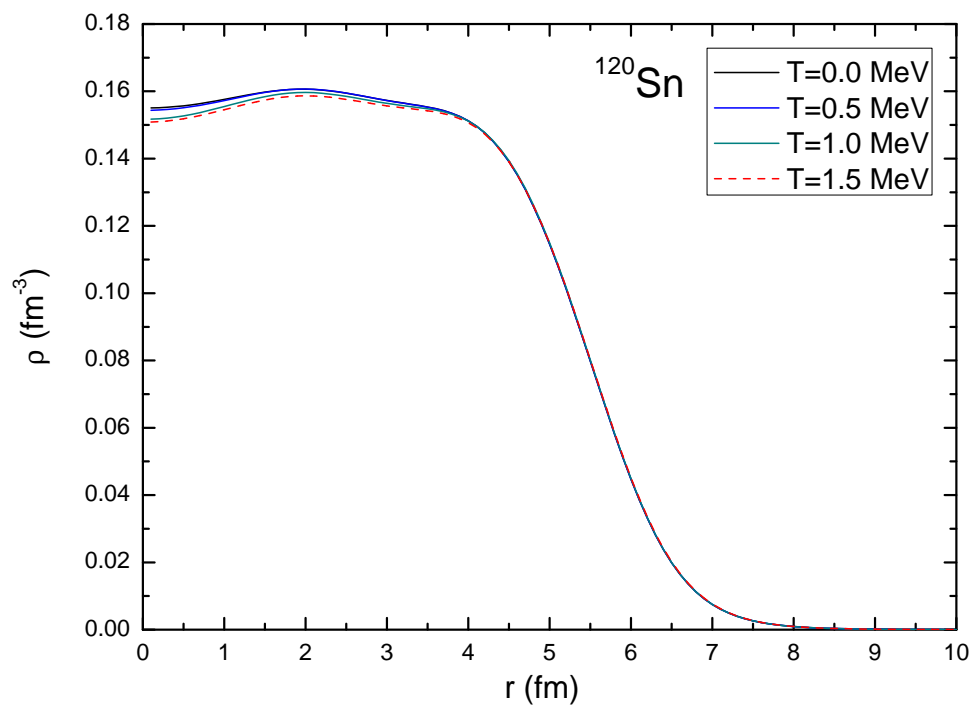

Figure 7: Change of the total density with respect to the temperature in ${ }^{120} \mathrm{Sn}$.

The changes in the effective masses depend on the density but not explicitly on the temperature. Therefore, the temperature dependence of the effective masses come indirectly from the temperature dependence of the density. (Eq.(19)). The total density is displayed in Fig. 7. In the center of the nucleus, the total density decreases with increasing temperature. Therefore the effective mass is expected to increase with increasing temperature, but there is also the effect of the isospin dependent density in Eq.(19): in the right part of Fig. 6, we display the change of the neutron and proton densities with respect to the temperature. The effect of temperature on the densities are pronounced above $\mathrm{T}=1.0 \mathrm{MeV}$ i.e., above critical temperature. After $\mathrm{T}=1.0 \mathrm{MeV}$, the neutron density decreases and the proton density increases in the interior of the nuclei. The decrease of the neutron density in the interior of the nuclei is directly linked to by the population of levels above the Fermi level, and depopulation of levels 
below (see Fig. 3), including the neutron $3 s_{1 / 2}$ state which contributes to the center of the nucleus. As a result, the neutron (proton) effective mass $\left(m_{q}^{*} / m\right)$ at $\mathrm{r}=0$ decreases (increases) after the critical temperature. The present results provide an accurate view of the temperature effects on the effective mass in nuclei. In order to provide a general trend for the behavior of the effective k-mass, we performed same calculations with other Skyrme interactions: SLy5, SIII and $\mathrm{SkM}^{*}$ and also with other nuclei. The results reveal that the behavior of the effective k-mass depends on the interaction used and nuclei considered. The kmass can either increase or decrease, however, the change is small and ranging between $0.15 \%$ and $1.1 \%$ from $\mathrm{T}=0 \mathrm{MeV}$ to $\mathrm{T}=1.5 \mathrm{MeV}$. Although in most of the calculations temperature dependence of the k-mass is small and taken as constant at first order, our results show that the temperature dependence of the k-mass should not be ignored in the case of an accurate study of the total effective mass at high temperatures.

\section{Conclusion}

In summary, we have investigated the pairing properties of different nuclei within the finite temperature HF+BCS framework with Skyrme interactions. The relation between the critical temperature and the pairing gap value at zero temperature generally obeys the $T_{c} \cong 0.57 \Delta_{T=0}$ equation, depending on the energy difference between the last occupied and first unoccupied states and the interaction used. The temperature dependence of the neutron skin is also investigated with different nuclei. A substantial increase in the proton and neutron radius of neutron rich nuclei is obtained above the critical temperatures. However for less-neutron rich nuclei, the increase in the proton radius cancels out the rapid increase of the neutron skin radius. The changes in the neutron skin is mainly due to the effect of temperature on the occupation probabilities of the singleparticle states around the Fermi level. High temperature causes the occupation of the unoccupied levels which is leading an enhancement in the proton and neutron radius.

A correlation is obtained between the effective mass of Skyrme energy density functionals and the critical temperature of ${ }^{120} \mathrm{Sn}$ nucleus, relating $m^{*}, \mathrm{~T}_{c}$ and $\Delta(T=0)$. It has been shown that with the enhancement in the effective mass, the level densities around the Fermi level and pairing gap increase which eventually leads an increase in the critical temperature. However this correlation depends on the pairing prescription. Temperature dependence of the effective k-masses of protons and neutrons are also investigated. Due to the changes in the proton and neutron densities, the effective nucleon masses show rather different fine behavior before and after the critical temperature, which is a new feature. 


\section{References}

[1] M. Sano and S. Yamasaki, Prog. Theor. Phys. 29397 (1963).

[2] A. L. Goodman, Nucl. Phys. A 35230 (1981).

[3] D. Gambacurta, D. Lacroix and N. Sandulescu, Phys. Rev. C 88034324 (2013).

[4] A. F. Fantina, J. Margueron, P. Donati and P. M. Pizzochero, J. Phys. G: Nucl. Part. Phys. 38025101 (2011).

[5] A. L. Goodman, Phys. Rev. C 341942 (1986).

[6] J. L. Egido and P. Ring, J. Phys. G: Nucl. Part. Phys. 191 (1993).

[7] V. Martin, J. L. Egido and L. M. Robledo, Phys. Rev. C 68034327 (2003).

[8] E. Khan, N. Van Giai and M. Grasso, Nucl. Phys. A 731311 (2004).

[9] J. Margueron and E. Khan, Phys. Rev. C 86065801 (2012).

[10] E. Khan, N. Van Giai and N. Sandulescu, Nucl. Phys. A 78994 (2007).

[11] Y. F. Niu, Z. M. Niu, N. Paar, D. Vretenar, G. H. Wang, J. S. Bai and J. Meng, Phys. Rev. C 88034308 (2013).

[12] A. Schiller, A. Bjerve, M. Guttormsen, M. Hjorth-Jensen, F. Ingebretsen, E. Melby, S. Messelt, J. Rekstad, S. Siem and S. W. Ødegård, Phys. Rev. C 63021306 (2001).

[13] E. Melby, M. Guttormsen, J. Rekstad, A. Schiller, S. Siem and A. Voinov, Phys. Rev. C 63044309 (2001).

[14] N. Sandulescu, Phys. Rev. C 70025801 (2004).

[15] C. Monrozeau, J. Margueron and N. Sandulescu, Phys. Rev. C 75065807 (2007).

[16] A. F. Fantina, PhD thesis, Universitè Paris Sud and Università degli Studi di Milano (2010).

[17] P. Donati, P. M. Pizzochero, P. F. Bortignon and R. A. Broglia, Phys. Rev. Lett. 722835 (1994).

[18] C. Mahaux, P. F. Bortignon, R. A. Broglia and C. H. Dasso, Phys. Rep. 1201 (1985).

[19] A. F. Fantina, P. Donati and P. M. Pizzochero, Phys. Lett. B 676140 (2009).

[20] D. J. Dean, K. Langanke and J. M. Sampaio, Phys. Rev. C 66045802 (2002). 
[21] P. Ring and P. Schuck, The Nuclear Many-Body Problem (Springer-Verlag, Heidelberg, 1980).

[22] G. F. Bertsch and H. Esbensen, Ann. Phys. (NY) 209, 327 (1991).

[23] J. Dobaczewski, H. Flocard and J. Treiner, Nucl. Phys. A 422103 (1984).

[24] M. Matsuo, Nucl. Phys. A 696371 (2001).

[25] P. Bonche, S. Levit and V. Vautherin, Nucl. Phys. A 42895 (1984).

[26] J. L. Egido, L. M. Robledo and V. Martin, Phys. Rev. Lett. 8526 (2000).

[27] E. Chabanat, P. Bonche, P. Haensel, J. Meyer and R. Schaeffer, Nucl. Phys. A 635231 (1998).

[28] N. Van Giai and H. Sagawa, Phys. Lett. B 106379 (1981).

[29] M. Beiner, H. Flocard, N. Van Giai and P. Quentin, Nucl. Phys. A 23829 (1975).

[30] J. Bartel, P. Quentin, M. Brack, C. Guet and H.-B. Håkansson, Nucl. Phys. A 38679 (1982).

[31] J. Dobaczewski, W. Nazarewicz, T. R. Werner, J. F. Berger, C. R. Chinn, and J. Dechargè, Phys. Rev. C 532809 (1996).

[32] D. Vautherin and D. M. Brink, Phys. Rev. C 5626 (1972).

[33] R.W. Hasse and P. Schuck, Phys. Lett. B. 179313 (1986).

[34] P. F. Bortignon and C. H. Dasso, Phys. Lett. B 189381 (1987). 